\title{
Effects of Salvia hispanica L. Seeds (Azpro) on Cardiovascular Risk Factors in Patients with Type 2 Diabetes - A Retrospective Observational Study
}

\author{
Dr. Piyush Desai ${ }^{1}$, Divyanshu Prajapati ${ }^{2}$ \\ ${ }^{1}$ Endocrinologist, Advanced Diabetes Centre, Surat, India. \\ ${ }^{2}$ Department of Medical Affairs, Azkka, Ahmedabad, India \\ Corresponding Author: Dr. Piyush Desai
}

\begin{abstract}
Background: India is the diabetes capital of the world. Despite several pharmacological options, almost $70 \%$ of patients present with poor $\mathrm{HbA} 1 \mathrm{c}$ control. Lifestyle modifications are one of the most important interventions for such chronic metabolic disorders. Therefore, adding a Lifestyle Modifier, a dietary approach collectively utilizing efficacious nutrients (natural source of very high dietary fiber, omega-3, proteins, minerals, and vitamins) while maximizing adherence is the key to the management of type 2 diabetes mellitus (T2DM).

Methodology: Patients were enrolled by adding Salvia hispanica L. Seeds (Azpro) along with standard medications to evaluate outcomes on risk factors in patients of T2DM. The objective was to determine the role of Salvia hispanica L. (Azpro), as an adjunct to conventional treatment in the reduction of major and emerging cardiovascular risk factors in individuals with type 2 diabetes. A retrospective analysis was conducted on fifty patients' data available at Advanced Diabetes Centre, Surat, India. Salvia hispanica L. (Azpro) seeds were given $10 \mathrm{~g}$ twice a day for 3 months as an adjunct to the standard diabetes management therapy. HbA1c, body fat, visceral fat, BMI, and weight loss were calculated at three months.
\end{abstract}

Results: Consumption of Salvia hispanica L. (Azpro) seeds for three months in addition to the standard treatment led to a total $1.13 \mathrm{~kg}$ weight loss $(p=0.0008)$, and $\mathrm{HbA} 1 \mathrm{c}$ reduction by $0.58 \%(p=0.016)$.

Conclusion: This result affirms the role of Salvia hispanica L. seeds in the dietary management of diabetic patients with significant $(P=0.0008)$ weight loss \& overall metabolic management.

Keywords: Salvia hispanica L. (Azpro) seeds, Chia seeds, diabetes, type 2 diabetes, weight loss, HbA1c, BMI, total fat, visceral fat, cardiovascular risk, dietary fiber, omega 3 fatty acids

\section{INTRODUCTION}

In India, the estimated cases of diabetes in adults are 72.96 million. The prevalence of diabetes in urban areas ranges from $10.9 \%$ to $14.2 \%$ and in rural areas it was 3 to $7.8 \%$ among the population of age over 20 years, with a much higher prevalence among individuals aged over 50 years (INDIAB Study). ${ }^{[1]}$ It is estimated that around 35 percent of Impaired Glucose Tolerance (IGT) patients develop type 2 diabetes mellitus (T2DM) in India. ${ }^{[2]}$ There exist several therapeutic options for the management of T2DM, but optimal control remains a challenge. Adherence, cost of therapy, patient awareness, and out-ofpocket expenditures are major challenges for patients. Despite several pharmacological options, $70 \%$ of patients present with poor HbA1c control.

Diet plays an important role in the pathology of T2DM, where the quality of carbohydrates matters because it can influence the digestion rate and thus the blood glucose response. Diets low in fiber and high in simple carbohydrates that cause 
sudden increases in blood sugar may increase the risk of developing type 2 Diabetes. ${ }^{[3]}$ Dietary factors or a balanced diet are very important in the management and prevention of type 2 diabetes. ${ }^{[4]}$ Universally, diabetes guidelines recommend nutritional and physical activity lifestyle changes as the first step. A high intake of dietary fiber showed an inverse correlation with T2DM. ${ }^{[5]}$ An alternative as safe as the right choice of food is the need of the hour. Lifestyle modifications being the most important factor for such chronic metabolic disorders like T2DM, it has always remained a challenge in implementation. A dietary approach collectively utilizing efficacious nutrients (natural source of high dietary fiber, omega-3, proteins, minerals, and vitamins) while maximizing adherence is the key to the management of T2DM. It is well known that dietary fiber may promote weight loss, enhances lipid profile and controls blood glucose, and reduces blood pressure. ${ }^{[6]}$ Dietary fibers can lead to weight loss by delaying gastric emptying and increasing the secretion of intestinal hormones which promote early satiety \& slow absorption of glucose. Leading organizations like American Heart Association and World Health Organization recommend lifestyle interventions like physical activity, maintaining a healthy weight, increasing the intake of fruits, vegetables, and whole grains, eliminating trans-fats, decreasing saturated fats, and increasing polyunsaturated fats (PUFA) consumption. ${ }^{[7]}$ PUFA, especially Omega-3 fatty acid-rich foods help in weight loss, improve plasma lipids, insulin resistance and decrease inflammation. ${ }^{[\mathbf{8 , 9}, 10]}$

Omega-3 fatty acid supplements significantly reduce $\mathrm{CV}$ events (death, nonfatal heart attacks, nonfatal strokes). Research suggests that Omega-3 fatty acids decrease the risk for arrhythmias, which can lead to sudden cardiac death, decrease the risk for thrombosis, which can lead to heart attack and stroke, decrease triglyceride and remnant lipoprotein levels, decrease the rate of growth of the atherosclerotic plaque, improve endothelial function, (slightly) lower blood pressure and reduce inflammatory responses. ${ }^{[11]}$ Evidence suggests consuming Salvia hispanica L. seeds can reduce systolic blood pressure, postprandial blood glucose and inflammation, due to its high content of dietary fiber and omega-3 fatty acid. There is growing evidence that whole grains may play an important role in the prevention of chronic diseases. Collective endorsement of whole grains by major health agencies around the world, including the Food and Drug Administration-approved health claim, is based on large prospective and epidemiological population studies that suggest a strong inverse relationship between increased consumption of wholegrain foods and reduced risk of diabetes and CVD. ${ }^{[12,13]}$ Salvia hispanica L., an ancient seed used as a food remedy by the Aztec civilization, is one of the highest whole food sources of dietary fiber and alpha-linolenic fatty acids (ALA), exceptionally rich in minerals, and a good source of protein. Salvia hispanica L. seeds improved major and emerging CVD risk factors in T2DM, suggesting a cardioprotective role by reduced postprandial glycemia when added to the daily meal. Salvia hispanica L. seeds represent the highest known whole-food source of dietary fiber and the Omega-3 polyunsaturated fatty acid (PUFA), alphalinolenic acid (ALA), in nature. Also, it is an exceptionally rich source of vegetable protein, calcium, magnesium, iron, and antioxidants. All these nutrients have been implicated in lowering CVD risks, and are naturally found in Salvia hispanica $\mathrm{L}$. seeds. ${ }^{[14]}$

Objectives: To investigate the effects of adding Salvia hispanica L. seeds (Azpro), to standard diabetes treatment, in the improvement of major and emerging cardiovascular risk factors in individuals with type 2 diabetes in Indian patients.

\section{MATERIALS AND METHODS}

A retrospective analysis was conducted on the patient pool data available 
at Advanced Diabetes Centre, Surat, India. Thirty-eight patients who were given Salvia hispanica L. (Azpro) seeds along with the standard diabetes treatment with inadequate glycemic control were considered. Participants were also instructed to maintain their prescribed therapy (recommended type and dose) of oral hypoglycemic, antihypertensive, or lipid-lowering medications.

These patients were asked to consume Salvia hispanica L. (Azpro) seeds $10 \mathrm{~g}$ twice a day for three months along with standard diabetes treatment. At the start of the study, baseline parameters including weight, BMI, visceral body fat, total body fat, and HbAlc were measured. These patients were regularly following up at the clinic and after three months of starting Salvia hispanica L. (Azpro) seeds, the above-mentioned parameters were repeated. The records of these patients were accessed retrospectively and relevant data was collected and analyzed.

Objectives: The primary outcome was a change in body weight and $\mathrm{HbA1c}$ at the end of three months. Secondary outcomes were a reduction in BMI, visceral body fat, and total body fat at the end of three months.

\section{RESULTS}

Out of the fifty patients considered for the investigation, thirty-eight patients completed the study and they were considered for the analysis. The baseline characteristics of these patients are mentioned in table 1 .

Table 1: Baseline demographics and illness characteristics of the patients

\begin{tabular}{|l|c|}
\hline \multicolumn{1}{|c|}{ Parameters } & Baseline (Mean \pm SD) \\
\hline Body weight $(\mathrm{kg}), \mathrm{n}=38$ & $85.34 \pm 16.02$ \\
\hline Body fat $(\%), \mathrm{n}=36$ & $34.79 \pm 7.5$ \\
\hline Visceral fat $(\%), \mathrm{n}=36$ & $16.17 \pm 5.06$ \\
\hline HbA1c $(\%), \mathrm{n}=38$ & $8.0 \pm 1.6$ \\
\hline BMI $\left(\mathrm{kg} / \mathrm{m}^{2}\right), \mathrm{n}=38$ & $30.56 \pm 5.75$ \\
\hline
\end{tabular}

SD(standard deviation), Body fat was not available for 2 patients out of the 38 patients considered.

Table 2: Results after three months of Salvia hispanica L. (Azpro) seeds as an adjunct to standard of care treatment

\begin{tabular}{|l|c|c|c|c|c|}
\hline \multicolumn{1}{|c|}{ Parameters } & Baseline & Follow-up & Absolute change from baseline & 95\% CI & P-value \\
\hline Bodyweight $(\mathrm{Kg}), \mathrm{n}=38$ & $85.34 \pm 16.02$ & $84.21 \pm 15.77$ & -1.132 & -1.757 to -0.506 & 0.0008 \\
\hline HbA1c $(\%), \mathrm{n}=38$ & $8.0 \pm 1.6$ & $7.4 \pm 1.06$ & -0.584 & -1.057 to -0.112 & 0.016 \\
\hline BMI $\left(\mathrm{kg} / \mathrm{m}^{2}\right), \mathrm{n}=38$ & $30.56 \pm 5.75$ & $30.26 \pm 5.64$ & -0.297 & -0.557 to -0.037 & 0.026 \\
\hline
\end{tabular}

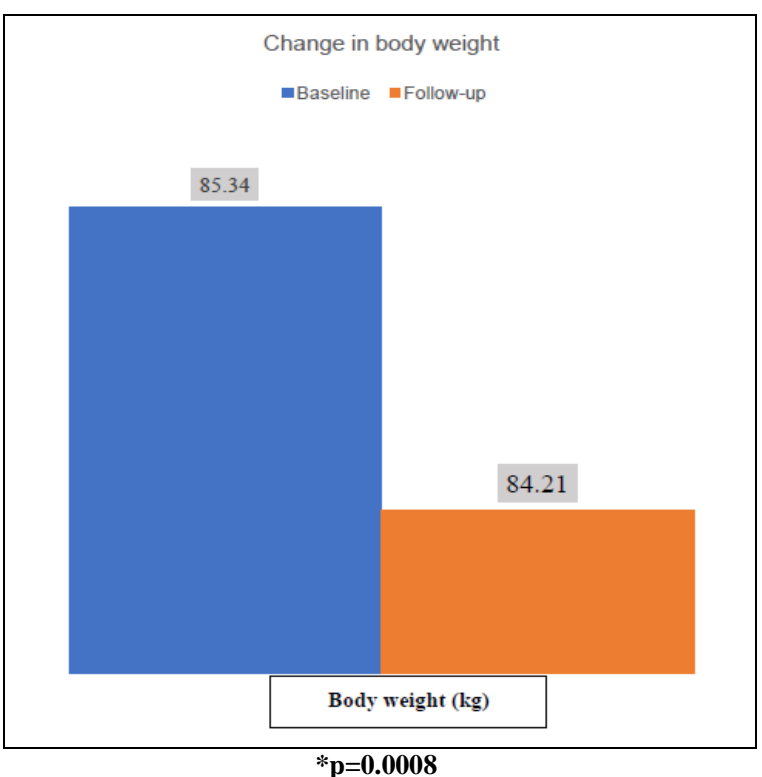

Fig. 1: Change in body weight after three months of Salvia hispanica L. (Azpro) seeds as an adjunct to standard diabetes treatment.

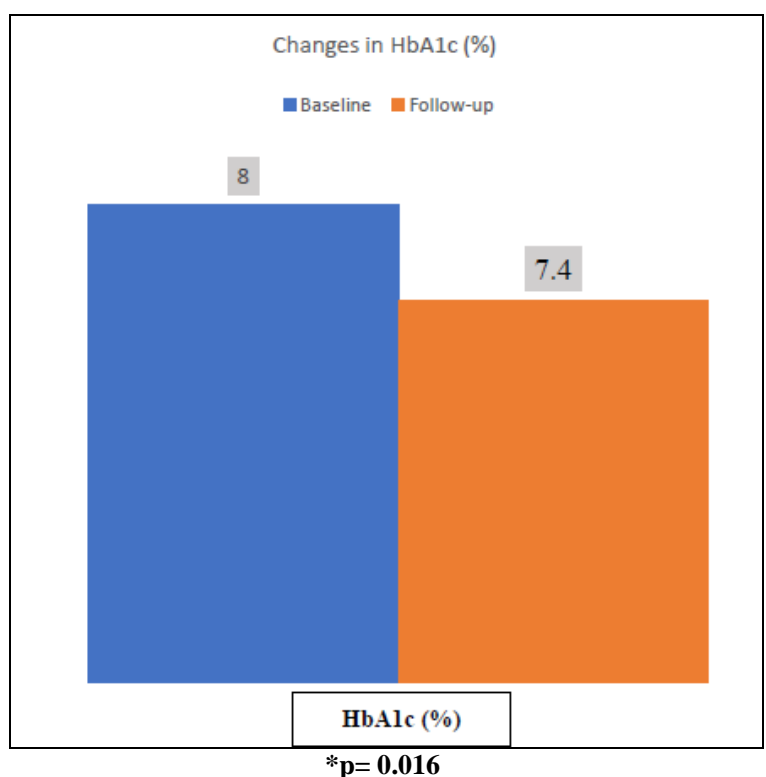

Fig. 2: Change in HbA1c (\%) after three months of Salvia hispanica L. (Azpro) seeds as an adjunct to standard diabetes treatment. 
Consumption of Salvia hispanica L. (Azpro) seeds for three months in addition to the standard treatment led to an average $1.13 \mathrm{~kg}$ weight loss (from $85.34 \mathrm{~kg}$ at baseline to $84.21 \mathrm{~kg}$ after 3 months; $p=0.0008$ ) (Fig.1), while HbA1c was reduced by $0.58 \%$ (from $8.0 \%$ to $7.4 \%$; $p=0.016$ ) (Fig.2).

The change in the secondary outcome was average reduction in BMI by $0.29 \mathrm{~kg} / \mathrm{m}^{2}$ (from $30.56 \%$ to $30.26 \%$; $p=0.026$ ) (Fig.3). All outcomes measured are presented in Table 2.

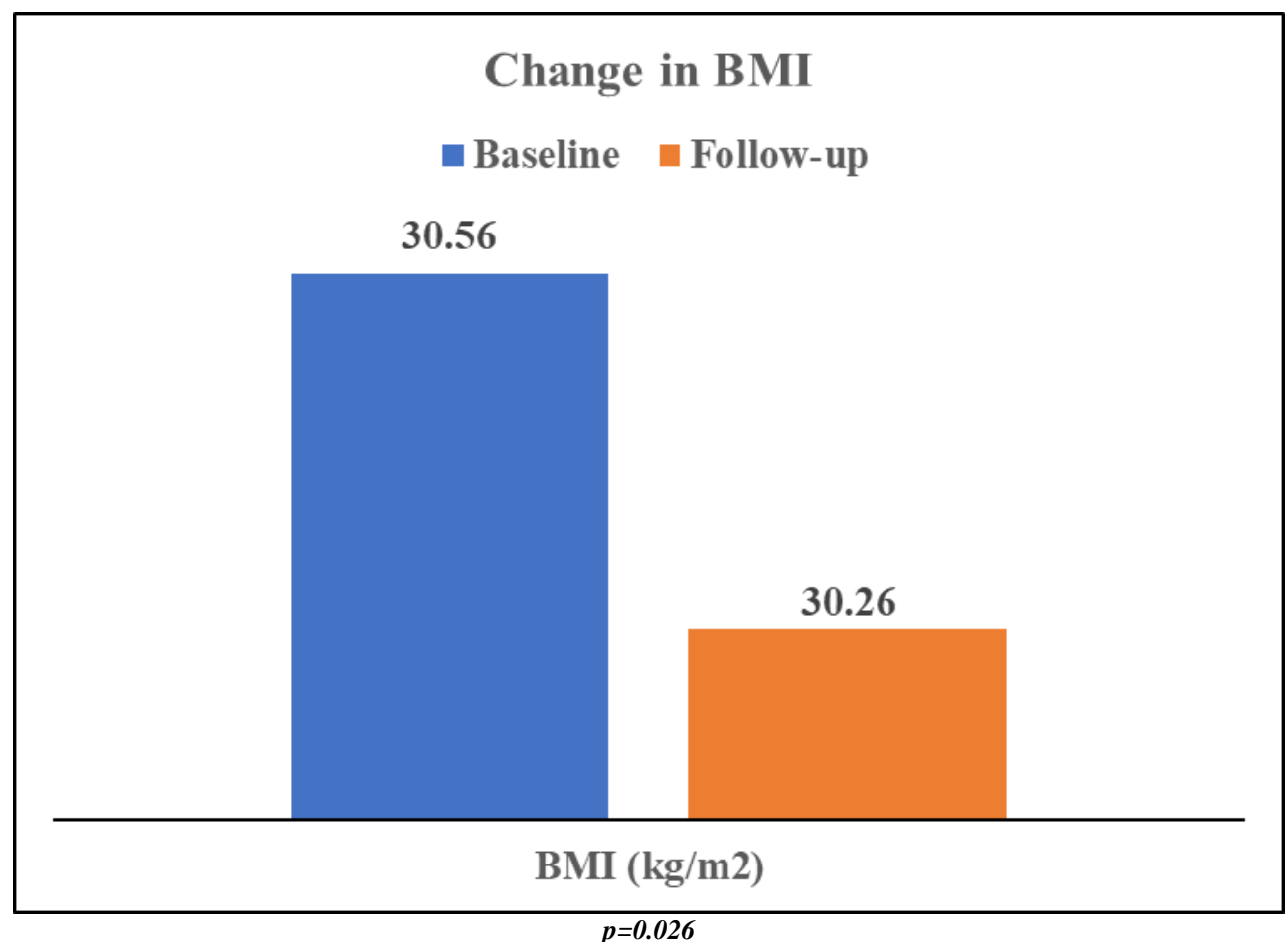

Fig. 3: Changes in BMI $\left(\mathrm{kg} / \mathrm{m}^{2}\right)$ after three months of Salvia hispanica L. (Azpro) seeds as an adjunct to standard diabetes treatment.

\section{DISCUSSION}

Salvia hispanica L. seeds are commonly known as Chia, which are edible seeds that come from the desert plant grown in Mexico dating back to Mayan and Aztec cultures. The meaning of the word "Chia" is strength, and they use these tiny black and white seeds as an energy booster. Salvia hispanica L. seeds contain healthy omega-3 fatty acids, carbohydrates, protein, fiber, antioxidants, and calcium. ${ }^{[15,16]}$ Salvia hispanica L. seeds are an unprocessed, whole-grain food that can be absorbed by the body as seeds (unlike flaxseeds). One ounce of chia seeds (i.e., 2 tablespoons) contains 139 calories, 12 grams carbohydrates out of which 11 grams are Dietary fiber 4 grams of protein, 9 grams fat, plus vitamins and minerals. ${ }^{[17]}$ The tasteneutral Salvia hispanica L. seeds make them easy to add to foods and beverages. They are often used with cereal, sauces, vegetables, rice dishes, or yogurt or mixed into drinks and baked goods. ${ }^{[10]}$ Increasing the intake of dietary fiber is known to have a favourable effect on overall metabolic health. Fiber-rich foods contain complex carbohydrates that are resistant to digestion and thereby reduce glucose absorption and insulin secretion. ${ }^{[18,19]}$ Diabetes patients when added Salvia hispanica L. seeds have shown to be benefitted from a favourable metabolic profile. Results from the present investigation revealed that the three-month addition of Salvia hispanica L. (Azpro) seeds to standard diabetes management, resulted in small, but significant, weight loss in patients with T2DM and a favorable HbA1c profile. Even a small loss in weight with a reduction in visceral obesity 
represents a clinically important achievement as weight management in T2DM is essentially challenging. Diabetes patients are mostly obese and these patients not only showed a small but significant weight loss but also HbA1c reduced with Salvia hispanica L. (Azpro) seeds.

Yao B. reported in the meta-analysis of 17 prospective cohort studies reported that there exists an inverse relationship between dietary intake and risk of T2DM. ${ }^{[20]}$

Earlier, Nieman et al. did not report weight loss with Salvia hispanica L. seeds. ${ }^{[21]}$ Differences in findings may be attributed to methodological variances, such as study design, non-diabetic population selection, and study material standardization. Salvia hispanica L. (Azpro) seeds in our study were given as an adjunct to the standard of care for the management of diabetes. Visceral fat is associated with greater mortality for any given BMI category. ${ }^{[22]}$ A decline in visceral adiposity induces adipokine secretion, such as adiponectin, and reduces inflammatory factors like high sensitivity C-reactive protein (hs-CRP), which are both suggested as surrogate cardiovascular markers in overweight and obese individuals.

The precise mechanism of action by which Salvia hispanica L. seeds promotes weight loss and improves obesity-related risk factors is unknown. Chia seeds are rich in omega-3-fatty acid, dietary fiber, protein, minerals, and a level of antioxidants which may act individually or together to demonstrate benefits. Numerous studies have shown that the ingestion of fiber can mitigate hunger, reduce postprandial glycemia, and promote weight loss. Dietary fiber has a proven role in reducing chronic inflammation, producing very small but significant reductions in hs-CRP of 0.37 $\mathrm{mg} / \mathrm{L}$ in obese populations. ${ }^{[23]}$

Overall, the investigation in Indian patients showed that patients with T2DM, when supplemented with Salvia hispanica L. (Azpro) seeds despite standard medical management, achieved its primary outcomes i.e., improvement in $\mathrm{HbAlc}$ and weight loss.

\section{CONCLUSION}

The investigation has shown that Salvia hispanica L. (Azpro) seeds when supplemented at a dose of $10 \mathrm{~g}$ twice daily in patients with T2DM who were on standard diabetes management were found to be benefitted. Not only the glycemic control improved, but it also led to the improvement in weight loss.

The role of Salvia hispanica L. (Azpro) seeds in T2DM is favourable with a statistically significant weight loss, BMI, and $\mathrm{HbAlc}$ were decreased. The overall metabolic management with Salvia hispanica L. (Azpro) seeds as an adjunct was decent in T2DM patients.

\section{Limitations and prospects}

- More multi-centric trials with a larger sample size are required to observe the beneficial effects of Salvia hispanica L. seeds in the management of diabetic patients.

- The given study was conducted retrospectively; prospective interventional trials can be carried out in the Indian population to achieve clinical outcomes and risk factor reduction using chia seeds.

- Instead of a before-after observational trial, a comparative study involving a control group can produce more substantial evidence. A randomized, controlled trial, with proper blinding techniques, is required to establish the Salvia hispanica L. seeds' benefits in CVS risk reduction in diabetic patients.

Conflict of Interest: None.

Acknowledgment: We acknowledge the support of Dr. Piyush Desai (Endocrinologist) for conducting the retrospective observational study in real world practice.

\section{Source of Funding: None}

Ethical Approval: Approved 


\section{REFERENCES}

1. Anjana RM, Deepa M, Pradeepa R, et al. Prevalence of diabetes and prediabetes in 15 states of India: results from the ICMRINDIAB population-based cross-sectional study [published correction appears in Lancet Diabetes Endocrinol. 2017 Aug;5(8): e5]. Lancet Diabetes Endocrinol. 2017;5 (8):585-596. doi:10.1016/S2213-8587(17)30174-2.

2. Editor. Diabetes in India.[Internet]. 2019 (accessed sep 2020). Available from: https://www.diabetes.co.uk/globaldiabetes/ diabetes-in-india.html.

3. Schulze MB, Liu S, Rimm EB, Manson JE, Willett WC, Hu FB. Glycemic index, glycemic load, and dietary fiber intake and incidence of type 2 diabetes in younger and middle-aged women. Am J Clin Nutr. 2004; 80(2):348-356. doi:10.1093/ajen/80.2.348.

4. Forouhi NG, Misra A, Mohan V, Taylor R, Yancy W. Dietary and nutritional approaches for prevention and management of type 2 diabetes. BMJ. 2018;361: k2234. Published 2018 Jun 13. doi:10.1136/bmj.k2234.

5. Mohan V, Radhika G, Sathya RM, Tamil SR, Ganesan A, Sudha V. Dietary carbohydrates, glycaemic load, food groups and newly detected type 2 diabetes among urban Asian Indian population in Chennai, India (Chennai Urban Rural Epidemiology Study 59) [published correction appears in $\mathrm{Br} \mathrm{J}$ Nutr. 2010 Jun;103(12):1851-2]. Br J Nutr.2009; 102(10):1498-1506. doi:10.1017/S0007114509990468.

6. Lattimer JM, Haub MD. Effects of Dietary Fiber and Its Components on Metabolic Health. Nutrients 2010; 2:12661289

7. Samuelson G. Global strategy on diet, physical activity and health. Food Nutr Res. 2004;48(2):57. http://dx.doi.org/10.3402/fnr.v48i2.1503.

8. Zhang $\mathrm{Y}$, Liu $\mathrm{W}$, Zhao $\mathrm{T}$, Tian $\mathrm{H}$. Efficacy of omega-3 polyunsaturated fatty acids supplementation in managing overweight and obesity: A meta-analysis of randomized clinical trials. J Nutr Health Aging. 2017; 21(2):187-192.
9. Yanai H, Masui Y, Katsuyama H, et al. An Improvement of Cardiovascular Risk Factors by Omega-3 Polyunsaturated Fatty Acids. Jounral Clin Med Res. 2018;10(4): 281-289.

10. Heilbrononn L, Campbell L. Adipose Tissue Macrophages, Low Grade Inflammation and Insulin Resistance in Human Obesity. Curr PharmDes.2013; 14(12):1225-1230. doi:10.2174/138161208784246153.

11. Kris-Etherton PM, Harris WS, Appel LJ; AHA Nutrition Committee. American Heart Association. Omega-3 fatty acids and cardiovascular disease: new recommendations from the American Heart Association. Arterioscler Thromb Vasc Biol.2003;23(2):151-152. doi:10.1161/01.atv.0000057393.97337.ae

12. U.S. Food and Drug Administration. 2020. Health Claim Notification For Whole Grain Foods. [online] Available at: $<$ https://www.fda.gov/food/food-labelingnutrition/health-claim-notification-wholegrain-foods $>$ [Accessed 4 September 2020].

13. Richardson DP. Wholegrain health claims in Europe. Proc Nutr Soc. 2003;62(1): 161-169. doi:10.1079/pns2002226.

14. Valdivia-Lopez MA, Tecante A. Chia (Salvia hispanica): a review of native mexican seed and its nutritional and functional properties. Adv Food Nutr Res 2015;75:53e75.

15. Suri, S., Passi, J. S., Goyat, J. Chia Seed (Salvia Hispanica L.)-A New Age Functional Food. 4th International Conference on Recent Innovations in Science Engineering and Management. March 20, 2016.

16. Koh AS, Pan A, Wang R, Odegaard AO, Pereira MA, Yuan JM, Koh WP. The association between dietary omega-3 fatty acids and cardiovascular death: the Singapore Chinese Health Study. Eur J Prev Cardiol. 2015 Mar;22(3):364-72.

17. Zelman KM. Chia Seeds Nutrition \& Facts About Weight Loss Claims. WebMD. Available at: https://www.webmd.com/diet/features/trut h-about-chia\#1. Accessed September 4, 2020. 
Piyush Desai et.al. Effects of Salvia hispanica L. seeds (Azpro) on cardiovascular risk factors in patients with type 2 diabetes-A retrospective observational study

18. Trivedi B, Maniyar KT, Patel B. Effect of fibre diet (guar) on cholesterol, blood glucose and body weight. Int $\mathbf{J}$ Diabetes Dev Ctries. 1999;19:31-3.

19. Lattimer JM, Haub MD. Effects of dietary fiber and its components on metabolic health. Nutrients. 2010;2:1266-89

20. Yao B, Fang H, Xu W, Yan Y, Xu H, Liu $\mathrm{Y}$, et al. Dietary fiber intake and risk of type 2 diabetes: A dose-response analysis of prospective studies. Eur J Epidemiol. 2014; 29:79-88.

21. Nowson CA, Wattanapenpaiboon N, Pachett A. Low-sodium Dietary Approaches to Stop Hypertension-type diet including lean red meat lowers blood pressure in postmenopausal women. Nutr Res. 2009; $29 \quad$ (1):8-18. doi:10.1016/j.nutres.2008.12.002.
22. Sahakyan KR, Somers VK, RodriguezEscudero JP, et al. Normal-Weight Central Obesity: Implications for Total and Cardiovascular Mortality. Ann Intern Med. 2015;163(11):827-835. doi:10.7326/M14 2525.

23. Vuksan, V., Rogovik, A.L., Jovanovski, E. et al. Fiber facts: Benefits and recommendations for individuals with type 2 diabetes. Curr Diab Rep 2009;9: 405-411. https://doi.org/10.1007/s11892009-0062-1.

How to cite this article: Desai P, Prajapati D. Effects of Salvia hispanica L. seeds (Azpro) on cardiovascular risk factors in patients with type 2 diabetes- a retrospective observational study. International Journal of Science \& Healthcare Research. 2021; 6(4): 57-63. DOI: https://doi.org/10.52403/ijshr.20211009 